\title{
Uso conjunto de piezometría y trazadores químicos e isotópicos para identificar áreas de recarga en ambientes áridos: zona del abanico de Agrelo, provincia de Mendoza, Argentina
}

\author{
Pabla Natalia Tognoli(1), Daniel Emilio Martínez ${ }^{(2)}$ y Sandra Ibáñez ${ }^{(1)}$
}

\begin{abstract}
(1) Instituto de Medio Ambiente, Facultad de Ingeniería, Universidad Nacional de Cuyo. Centro Universitario, M5502JMA, Mendoza, Argentina. pablatognoli@yahoo.com; sandra.ibanez@ingenieria.uncu.edu.ar

(2) Instituto de Investigaciones Marinas y Costeras. CONICET. Inst. de Geología de Costas y del Cuaternario, CIC Prov. de Buenos Aires. Universidad Nacional de Mar del Plata. Funes 3350, C.P. (7600), Mar del Plata, Argentina.
\end{abstract} demarti@mdp.edu.ar

RESUMEN

Si bien el área del Abanico de Agrelo cuenta con numerosos estudios de carácter hidrogeológico por su importancia desde el punto de vista de la recarga del acuífero de Cuenca Norte de Mendoza, aún existen incertidumbres por resolver. El objetivo de este trabajo es incrementar la información disponible para actualizar y mejorar el modelo conceptual del acuífero, reconociendo las posibles zonas de recarga a partir del uso de piezometría y datos hidroquímicos e isotópicos. Para ello se realizó la recopilación de antecedentes y se redefinió la dinámica de flujo del acuífero estudiado. Sobre la base de estos antecedentes, se planificó y llevó a cabo un muestreo hidroquímico e isotópico con el fin de corroborar la dinámica hidrológica. Los resultados indican que el flujo predominante del sistema tiene sentido NO-SE y que, si bien el río Mendoza es la principal fuente de recarga, podrían existir otras áreas de aporte. Los datos hidroquímicos corroboran la dinámica, mostrando un enriquecimiento paulatino de sales en el sentido del flujo. El patrón hidroquímico subterráneo es similar al del río, pero con valores de conductividad menores en la zona proximal, indicando posible mezcla de aguas a partir de otra fuente de recarga adicional. El modelo conceptual resultante considera que el sistema hidrogeológico del Abanico de Agrelo se extiende hacia ambas márgenes del río Mendoza y que el río no constituye un borde del sistema acuífero en sí, ya que funciona como una zona de recarga. Además, muestra la existencia de fuentes de recarga adicionales al río.

Palabras clave: Abanico de Agrelo Mendoza, hidrodinámica, hidroquímica, modelo conceptual hidrogeológico, recarga.

\section{Joint use of piezometric surfaces and chemical and isotopic tracers to identify recharge areas in arid environments: Agrelo fan area, Mendoza province, Argentina}

\begin{abstract}
Although there are numerous hydrogeological papers on the Abanico de Agrelo area due to its importance from the point of view of recharge to the Mendoza Northern Basin, many uncertainties still remain to be solved. The aim of this study is to obtain new data to update and improve the conceptual model of the aquifer. For this purpose, we have carried out a desktop review of the previous data and the aquifer dynamics have been redefined. Based on this information, a hydrochemical and isotopic sampling was carried out in order to confirm the hydrogeological dynamics. Our results indicate that the main flow of the aquifer is NW-SE and that although the river is the main source of recharge, there could be other input areas. The hydrochemical data confirm this dynamic, showing a gradual enrichment of salts in the flow direction. The hydrochemical underground pattern is similar to that of the river, but it shows lower conductivity values in the nearby areas, in-
\end{abstract}


dicating a probable mixture of water from another recharge source. The resulting conceptual model considers that the hydrogeological system of the Abanico de Agrelo extends to both banks of the Mendoza River and that the river is not an edge of the aquifer itself as it works as an indirect recharge area. The existence of additional recharge sources is also proposed in this paper.

Keywords: Agrelo Fan Mendoza, conceptual model, hydrochemistry, hydrodynamic, recharge.

\section{Introducción}

La provincia de Mendoza, situada en el centro oeste de la República Argentina, se encuentra en una zona de clima árido a semiárido. Estas zonas requieren un especial énfasis en la comprensión de los diferentes elementos del balance hidrológico en pos de diseñar políticas de manejo de los recursos hídricos. Para alcanzar ese objetivo es necesario considerar diferentes enfoques de manera integrada.

El Abanico de Agrelo en el sector norte de la Depresión del Carrizal (Giaccardi et al., 2018), reviste interés ya que corresponde a la principal zona de recarga del acuífero que alimenta al mayor núcleo poblacional y al principal oasis productivo de la provincia de Mendoza (Figura 1). Constituye además un sector de importante producción agrícola y actividad industrial, donde actualmente existe fuerte presión para la instalación de nuevos proyectos productivos que requie- ren del recurso hídrico para uso consuntivo. En el año 2003, se construyó sobre el Río Mendoza, aguas arriba de la zona estudiada, el Dique Potrerillos (Fig. 1). Estudios recientes fueron realizados aguas arriba de dicho dique (Massone et al., 2016), en las nacientes del Río Mendoza (Crespo et al., 2016), y en el este de la zona de estudio (Gómez et al., 2014). Se busca en este trabajo actualizar la información hidrogeológica básica para este nuevo contexto, estableciendo la dinámica de flujo sobre la base del uso integrado de estudios piezométricos y trazadores químicos e isotópicos para mejorar el modelo conceptual existente.

\section{Características de la zona}

El área de estudio pertenece al ambiente del Pedemonte Mendocino. Es un área deposicional limitada por los altos de la cerrillada Cacheuta-Tupungato, la Precordillera por el Oeste y la Cerrillada Lunlunta-Ca-
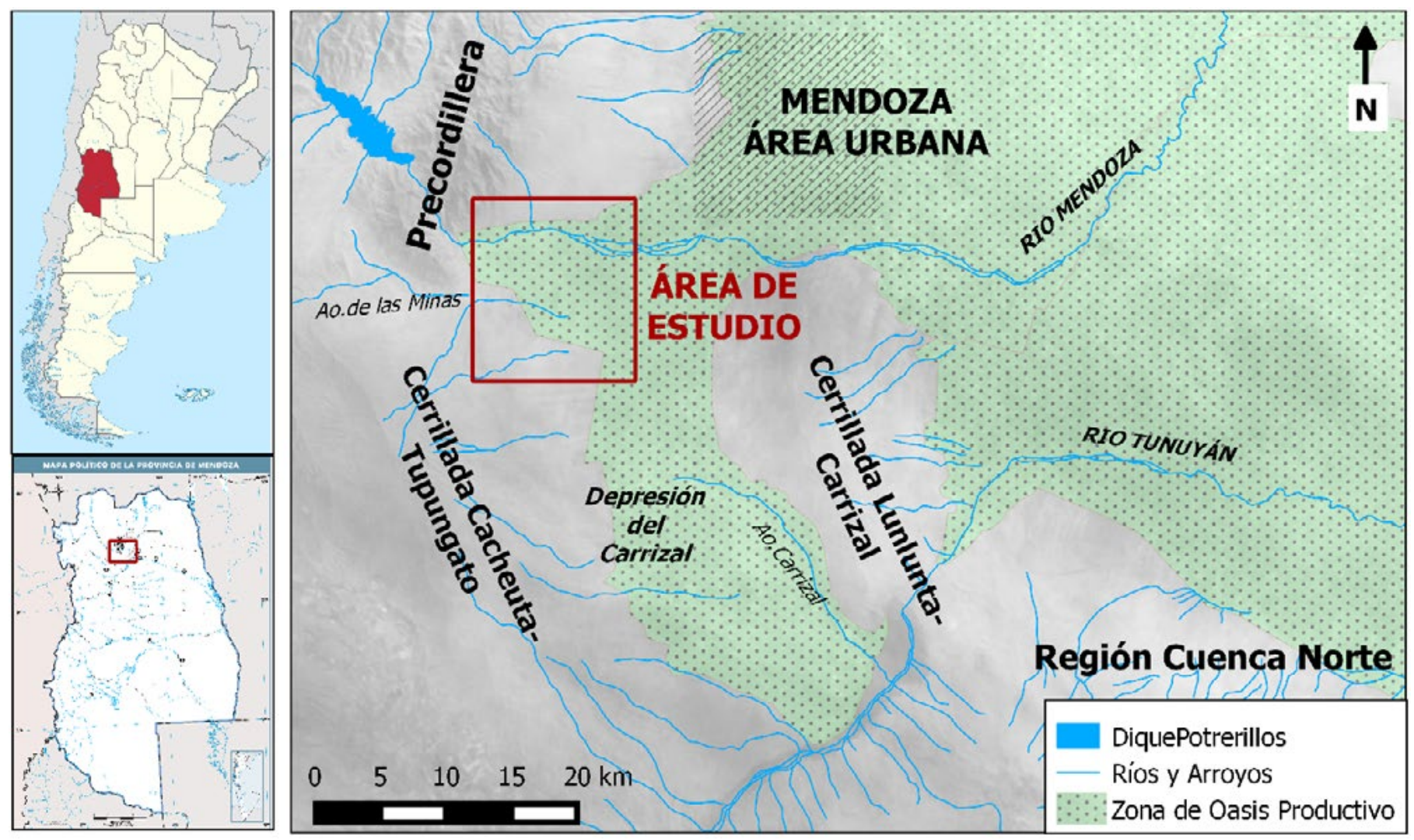

Figura 1. Ubicación regional. Figure 1. Regional location. 


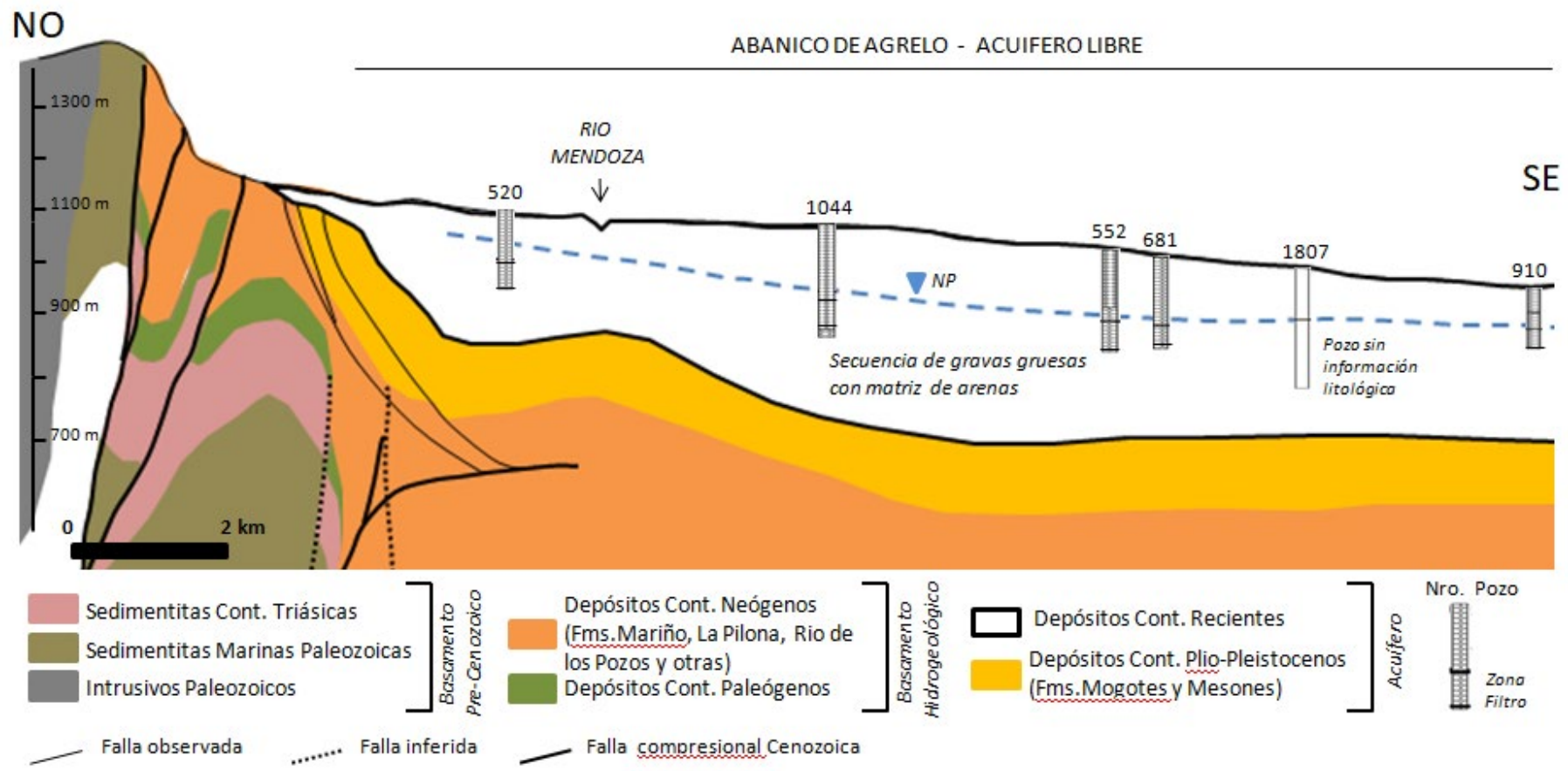

Figura 2. Sección estratigráfica e hidrogeológica NO-SE (estratigrafía modificada de Giambiagi et al, 2003). La traza de la sección se indica en la Figura 3.

Figure 2. Stratigraphic and hydrogeologic section NW-SE (stratigraphy modified from Giambiagi et al, 2003). The section is shown in Figure 3.

rrizal por el este. Está atravesada por el Río Mendoza el cual nace en la Cordillera de los Andes. Casi en su totalidad, su caudal proviene del derretimiento de la nieve y hielo. Las precipitaciones pluviales que se registran en su cuenca inferior no sobrepasan los 250 $\mathrm{mm}$ anuales y no influyen en los caudales del río. Tiene un derrame medio anual de aproximadamente $1.400 \mathrm{hm}^{3}$, con un valor de derrame para años secos de $860 \mathrm{hm}^{3}$ y para años húmedos de $2.300 \mathrm{hm}^{3}$. El módulo del río calculado para el período 1956/57-2012/13 es de aproximadamente $45 \mathrm{~m}^{3} / \mathrm{s}$ (Ibáñez et al., 2013). EI río cuenta con el embalse regulador, Potrerillos, que tiene una capacidad de $450 \mathrm{hm}^{3}$ y comenzó a funcionar a fines del año 2004.

El Abanico de Agrelo es un abanico aluvial típico que tiene su ápice en el Arroyo de las Minas y se extiende hacia el Este por aproximadamente $15 \mathrm{~km}$. En su borde Norte coalesce con los abanicos aluviales que descienden de la Precordillera y hacia el Sur presenta un borde erosivo. Tiene por lo menos $10 \mathrm{~km}$ de extensión en sentido N-S y $15 \mathrm{~km}$ O-E. Forma parte de la Depresión del Carrizal. Esta es un paleovalle, que en su mitad septentrional está relleno de depósitos cuaternarios de piedemonte, que forman el acuífero objeto de este trabajo.

Geológicamente la región está caracterizada por la presencia de un basamento paleozoico y permo-triásico, el relleno de una cuenca extensional triásica, una cobertura paleógena y neógena caracterizada por depósitos relacionados a la orogenia andina y por último depósitos cuaternarios aluviales asociados a ambientes pedemontanos y de llanuras aluviales. Los terrenos paleozoicos y permo-triásicos afloran en la Precordillera. Las rocas del Paleógeno, del Neógeno y del Cuaternario afloran en el resto del área y son de gran importancia en el aspecto hidrogeológico, ya que ellas constituyen la base hidrogeológica de la cuenca de agua subterránea (Fms. Mariño, La Pilona, Río de los Pozos) o bien son portadoras de los acuíferos explotables (Fms. Mogotes, Mesones y otros sedimentos cuaternarios) (Valero, 1993) (Figura 2).

Las características de los acuíferos de la zona se conocen a partir de las aproximadamente 180 captaciones de agua subterránea existentes. Estas alcanzan profundidades entre 100 y 280 metros, captando en casi todos los casos la parte superior del acuífero cuyo nivel freático se encuentra a profundidades superiores a 150 metros en el oeste y cercano a los 40 metros próximo al límite oriental. Se trata de un acuífero libre, granular y de gran espesor, que se aloja mayoritariamente en sedimentos cuaternarios gruesos (arenas y gravas) asociados a abanicos y planicies aluviales y secundariamente en sedimentos neógenos gruesos que presentan mayor grado de cementación o consolidación ( $\mathrm{Fm}$. Mogotes). El espesor total del acuífero en el área fue estimado en más de $450 \mathrm{~m}$ por Valero 
(1993) a través de estudios geofísicos, sin embargo la base del acuífero todavía no ha sido alcanzada por ninguna perforación. El mismo ha sido caracterizado como acuífero libre de alta permeabilidad (Foster y Garduño, 2005; Foster et al., 2018, Gómez et al., 2014). Se desconoce si existen niveles confinados en profundidad ya que los pozos más profundos no superan los $280 \mathrm{~m}$. Al este, algunos pozos presentan litologías con facies aluviales finas interidigitadas, que confieren ciertas características de semiconfinamiento. Incluso más hacia el este, fuera de la zona de estudio, se alcanzan niveles de confinamiento con comportamiento artesiano (Hernández y Martinis, 2006). A gran escala constituye un único sistema acuífero multicapa.

\section{Metodología}

La primera etapa del trabajo consistió en la recopilación y revisión de antecedentes y de datos de las perforaciones existentes. Las tareas de campo se iniciaron con un inventario de distintos puntos de agua (tanto superficiales como captaciones). Durante agosto y septiembre de 2016 se midieron niveles estáticos en 20 perforaciones y se tomaron muestras para análisis hidroquímicos en 21 sitios (Figura 3); en estos últimos se midieron "in situ" parámetros fisicoquímicos, como $\mathrm{pH}$, conductividad eléctrica (CE) y temperatura. Las muestras fueron analizadas en el laboratorio de Instituto de Hidrología de Llanuras de Azul (ILLHA), donde se realizaron las siguientes determinaciones: $\mathrm{pH}$ y $\mathrm{CE}$, al-

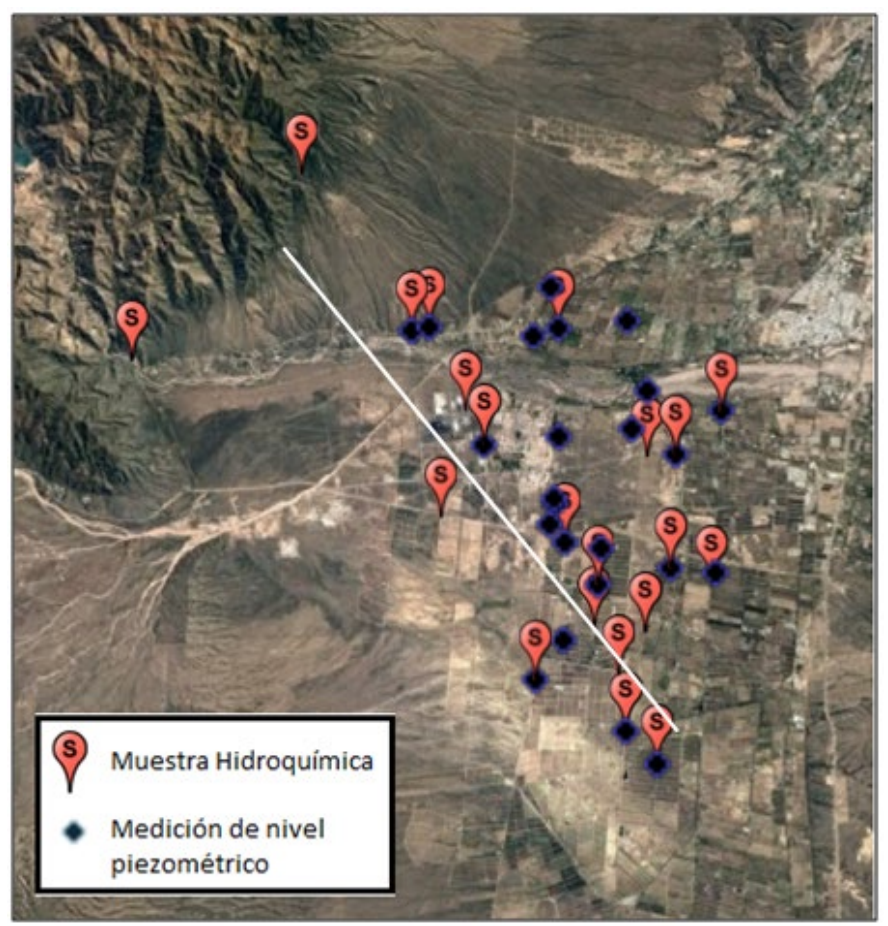

Figura 3. Sitios de muestreo hidroquímico y medición de nivel piezométrico. La sección de la Figura 2 se indica en blanco.

Figure 3. Hydrochemical sampling and piezometric level measurement sites. Section of Figure 2 is marked out in white. calinidad, dureza, aniones mayoritarios $\left(\mathrm{HCO}_{3}^{-} \mathrm{y} / \mathrm{o} \mathrm{CO}_{3}\right.$ $\left.{ }^{2}, \mathrm{SO}_{4}^{-2}, \mathrm{Cl}^{-}, \mathrm{NO}_{3}^{-}\right)$y cationes mayoritarios $\left(\mathrm{Na}^{+}, \mathrm{K}^{+}, \mathrm{Ca}^{+2}\right.$, $\mathrm{Mg}^{+2}$ ). Los cationes fueron analizados por espectrofotometría de Absorción Atómica de Llama (SM3111), mientras que los $\mathrm{HCO}_{3} / \mathrm{CO}_{3}{ }^{-2}$ se analizaron por valoración de ácido base (SM2320), el $\mathrm{SO}_{-}^{-2}$ por espectrofotometría uv-visible (EPA. Método 375.4 ), el $\mathrm{Cl}^{-}$por método argentométrico (SM4500-Cl-B) y el $\mathrm{NO}_{3}^{-}$por espectrometría ultravioleta selectiva (SM4500- $\mathrm{NO}_{3}{ }^{-\mathrm{B}}$ ). El muestreo incluyó 19 captaciones de agua subterránea, todas ellas con equipo de bombeo electrosumergible, lo que permitió purgar adecuadamente los pozos y muestrear con facilidad; 1 pequeño arroyo que drena agua de la vertiente La Crucesita, ubicada en el sector NO de la zona de estudio en la Precordillera y 1 muestra del río Mendoza en la zona del cañadón de Cacheuta, 250 metros antes de que el río desemboque en la zona de pedemonte. Los 19 pozos muestreados se seleccionaron de acuerdo a su distribución espacial dentro de la zona de estudio y considerando el mismo nivel de captación. Una vez recibidos los resultados de laboratorio, se procedió a estimar el error de balance iónico (Custodio y Llamas, 1983), el cual fue menor a $8 \%$ en 20 de los casos y cercano a $10 \%$ en 1 muestra.

Para la mayoría de las perforaciones analizadas se contó con datos de la secuencia estratigráfica atravesada y del diseño de las captaciones. Con datos de los ensayos de pozos, que poseen todas las perforaciones, se obtuvo el caudal específico y a partir de la formula empírica de Galofré (1966) se estimaron datos de transmisividad.

En el momento del muestreo hidroquímico, se tomaron muestras para análisis de isotopos estables: 9 de agua subterránea, 1 del río y 1 de la vertiente. Los análisis isotópicos se realizaron en el Laboratorio de Hidrología Isotópica del Instituto de Geología de Costas y del Cuaternario de la Universidad de Mar del Plata mediante espectroscopia láser (Lis et al. 2008), utilizando un analizador de isótopos de agua líquida 45-EP, de inyección automatizada, desarrollado por Los Gatos Research. Los resultados se expresaron como valores de desviación isotópica $\delta$ en pormil $(\% \circ)$, definidos como: $\delta \%=1000$ (Rs - Rp) / Rp; s es la muestra; $p$ es la referencia internacional; y $\mathrm{R}$ es la relación isotópica $\left({ }^{2} \mathrm{H} /{ }^{1} \mathrm{H},{ }^{18} \mathrm{O} /{ }^{16} \mathrm{O}\right)$. El estándar es el agua de media de océano de Viena (VSMOW; Gonfiantini 1978). Las incertidumbres analíticas fueron de $\pm 0,2 \%$ o para $\delta^{18} \mathrm{O}$ y de $\pm 2,0 \%$ o para $\delta^{2} \mathrm{H}$.

\section{Resultados}

\section{Dinámica de flujo y parámetros hidráulicos}

El principal acuífero es de tipo libre y presenta niveles estáticos entre 150 y 40 metros de profundidad. La piezometría muestra una dinámica de flujo con una dirección predominante en sentido NO-SE y una notoria divergencia del flujo en el sector nororiental y oriental (Figura 4.a). 

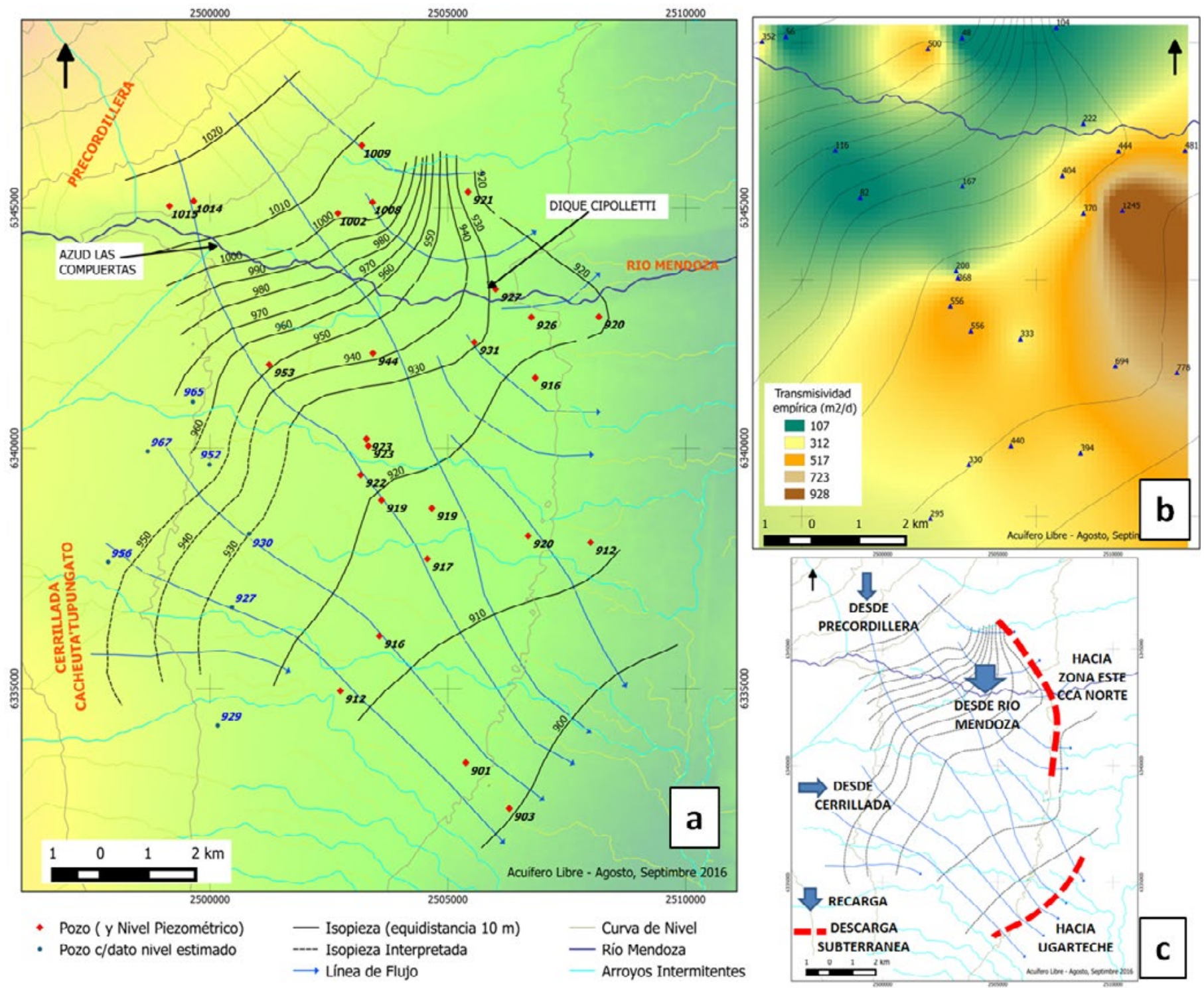

Figura 4. a) Piezometría y dinámica de flujo. b) Mapa de isotransmisividad (T en $\mathrm{m}^{2} / \mathrm{d}$ ) (raster); las curvas son las equipotenciales. c) Zonas de recarga y descarga y sentido de flujo.

Figure 4. a) Piezometric surface and flow dynamic. b) Transmissivity map ( $T$ in $\mathrm{m}^{2} / \mathrm{d}$ ) (raster) and equipotential lines c) Recharge and discharge zones, and flow lines.

La isopieza más elevada es la de 1.020 m s.n.m y se ubica en el extremo NO. La isopieza de menor altitud es la de 900 m s.n.m y se encuentra en el extremo SE. El gradiente hidráulico promedio en sentido NO-SE es de $7,5 \cdot 10^{-3}$. En el sector norte, donde se registran los gradientes hidráulicos más elevados, estudios previos (Valero, 1993) detallan para esa zona una elevación del basamento hidrogeológico, con predominancia de sedimentitas neógenas ( $\mathrm{Fm}$. Mogotes) frente a depósitos Cuaternarios. Estas sedimentitas presentan mayor cementación (menor conductividad hidráulica), explicando posiblemente los cambios de gradiente hidráulico. A partir de la isopieza $930 \mathrm{~m}$, el gradiente hidráulico disminuye hacia los valores más bajos encontrados $\left(3,3.10^{-3}\right)$, coincidiendo este hecho con una división del flujo subterráneo en tres direcciones principales: O-E y SO-NE en la margen norte del río Mendoza, NO-SE y O-E inmediatamente al sur del río y NO-SE en la zona distal. Otro rasgo muy importante que destacan las isopiezas es que, por debajo de la isopieza de $950 \mathrm{~m}$, el río parece tener un comportamiento influente más típico. Las isopiezas en el sector SO son una interpretación tentativa, ya que es una zona donde no se obtuvieron datos de campo. Para esta interpretación, se consideraron datos de niveles estáticos registrados al momento de la construcción de los pozos de la zona.

Para contar con datos de parámetros hidráulicos, 


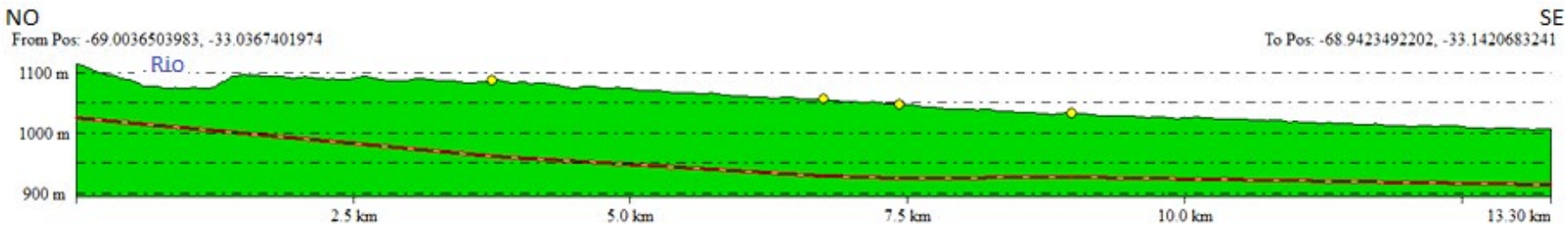

Figura 5. Sección topográfica a partir de datos DEM donde se muestra la superficie del nivel estático. Nótese la desconexión saturada entre la base del Río Mendoza y el techo del acuífero.

Figure 5. Topographic section based on DEM data showing the water table. Note the saturated disconnection between the base of the Mendoza River and the top of the aquifer.
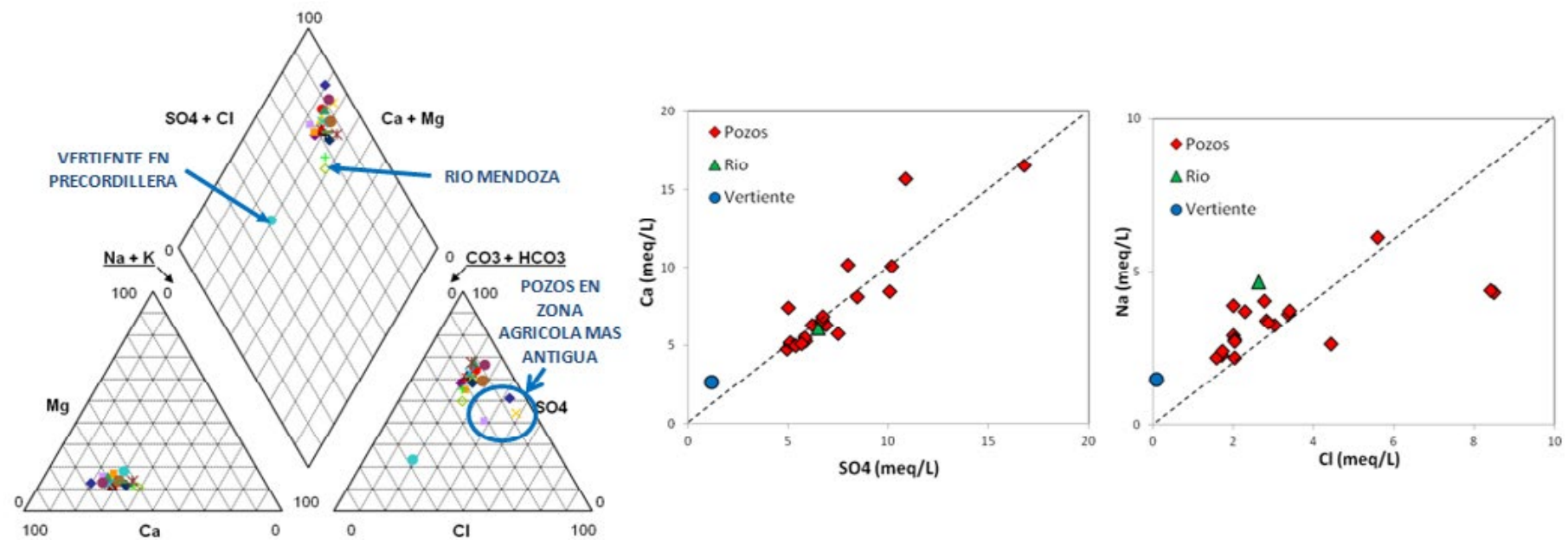

Figura 6. Diagrama Piper, correlación $\mathrm{SO}_{4}{ }^{2}-$ y $\mathrm{Ca}^{2+}(\mathrm{meq} / \mathrm{L})$ y correlación $\mathrm{Cl}^{-}$vs $\mathrm{Na}^{+}(\mathrm{meq} / \mathrm{L})$.

Figure 6. Piper diagram, $\mathrm{SO}_{4}{ }^{2}-\mathrm{vs} \mathrm{Ca}^{2+}(\mathrm{meq} / \mathrm{L})$ and $\mathrm{Cl}$ vs $\mathrm{Na}^{+}(\mathrm{meq} / \mathrm{L})$ correlation graphs.
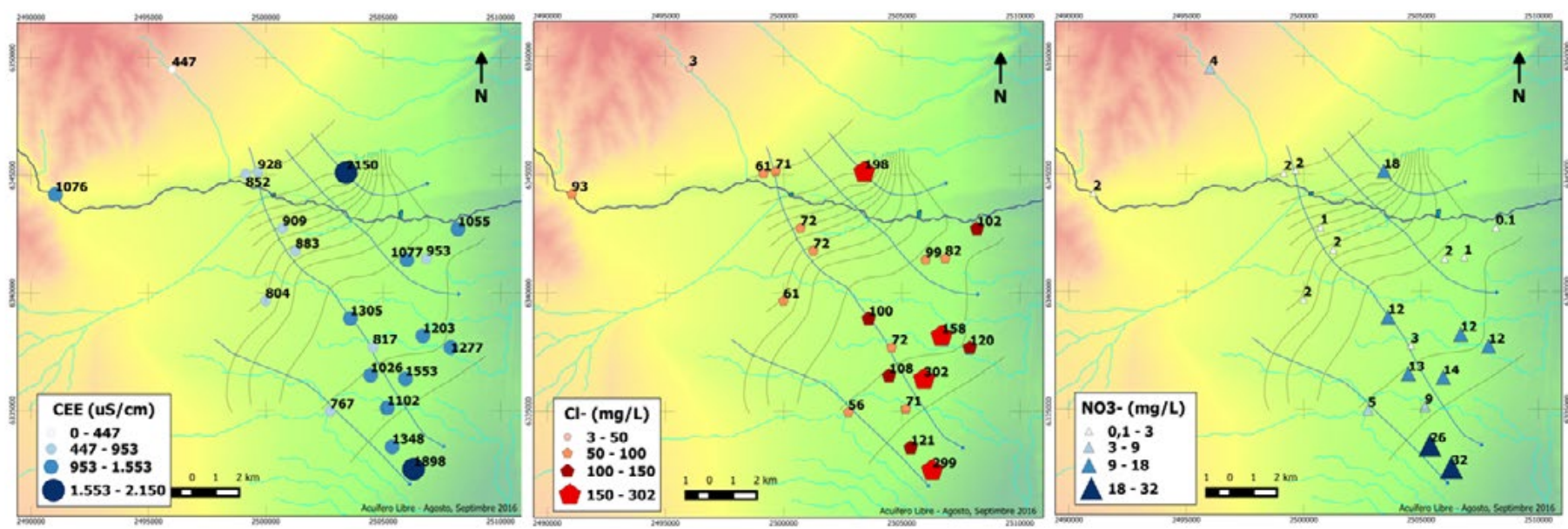

Figura 7. Distribución de $\mathrm{CE}(\mu \mathrm{S} / \mathrm{cm}), \mathrm{Cl}^{-}(\mathrm{mg} / \mathrm{L})$ y $\mathrm{NO}_{3^{-}}(\mathrm{mg} / \mathrm{L})$.

Figure 7. $\mathrm{CE}(\mu \mathrm{S} / \mathrm{cm}), \mathrm{Cl}(\mathrm{mg} / \mathrm{L})$ y $\mathrm{NO}_{3^{-}}(\mathrm{mg} / \mathrm{L})$ areal distribution.

se calculó la transmisividad ( $\mathrm{T}$ ) a partir de datos de ensayos de pozos (caudal específico) existentes en la información técnica de los pozos (Figura 4.b). Los valores de T obtenidos empíricamente varían entre 47 y $1.245 \mathrm{~m}^{2} / \mathrm{d}$. Los valores más bajos se ubican en la zona norte (en la margen izquierda del Río Mendoza), coincidiendo con la zona de mayor gradiente hidráulico.
Los valores más altos se localizan en el sector oriental y coinciden con la zona de menor gradiente. Entre ambos valores extremos hay fluctuaciones, pero en general la T aumenta en sentido NO-SE, coincidente además con un aumento en el espesor de sedimentos saturados (Valero, 1993). Los valores de T considerados correlacionan bien con la información generada 
a partir de la medición de niveles, reflejada en la piezometría. LaT calculada a partir del caudal específico puede ser un buen parámetro para interpretaciones de carácter relativo y zonificaciones.

Las zonas de recarga según este modelo de flujo son 3 (Figura 4.c): una en el extremo NO, desde la Precordillera, a partir de la infiltración de agua de manantiales y lluvia en la zona proximal de los abanicos aluviales al pie de la sierra; un segundo sector, aunque con incertidumbre, desde el Oeste con los aportes de las Cerrilladas de Cacheuta y Tupungato; y una tercera zona de recarga vinculada al río Mendoza. La descarga natural del acuífero se produce fuera de la zona de estudio.

La piezometría permitió definir la relación hidráulica entre el río y el acuífero, mostrando el carácter influente del primero. Si se compara el nivel piezométrico del acuífero en la zona cercana al río, con las cotas del río a lo largo de su recorrido, se observa una diferencia entre ambos que oscila en los 50-80 metros (las cotas del río se estimaron a partir de una imagen DEM); este hecho, sumado al comportamiento de río perdedor, clasifica la relación río acuífero como río influente, que recarga al acuífero de forma indirecta a través de una zona no saturada de importante espesor. (Figura 5).

\section{Hidroquímica}

La mayoría de las aguas muestreadas son de tipo sulfatada cálcica, con la excepción del arroyo-vertiente la Crucesita, en la Precordillera, que es bicarbonatada cálcica (Figura 6).

En los pozos cercanos a las márgenes del río (1.076 $\mu \mathrm{S} / \mathrm{cm})$, las aguas del acuífero mantienen las características de éste (tipo sulfatada-cálcica), aunque presentan valores de conductividad eléctrica (CE) algo menores (852-928 $\mu \mathrm{S} / \mathrm{cm}$ ) (Figura 7).

Esto también se repite en los pozos localizados hacia el extremo oeste $(804-909 \mu \mathrm{S} / \mathrm{cm})$. Esto podría dar indicios de la existencia de mezcla de aguas de recarga del propio río, con aguas provenientes de la zona de Precordillera $(447 \mu \mathrm{S} / \mathrm{cm})$ y de las Cerrilladas del oeste, las cuales están menos mineralizadas. En el acuífero, la CE aumenta en el sentido de las líneas de flujo; este aumento está relacionado principalmente a un proceso de disolución de sales aguas abajo, sobre todo de $\mathrm{CaSO}_{4}$, que está presente como yeso en los sedimentos cuaternarios. El yeso como fuente de iones $\mathrm{Ca}^{2+}$ y $\mathrm{SO}_{4}{ }^{2-}$ resulta claramente de la composición y solubilidad de este mineral (Elango and Kannan, 2007). La relación entre el aumento de estos iones y el aumento de CE presenta buena correlación. El aumento de CE se corresponde también con el aumento de $\mathrm{Cl}^{-}$y $\mathrm{Na}^{+}$en el sentido de flujo. El intercambio iónico entre $\mathrm{Ca}^{2+}$ y $\mathrm{Na}^{+}$es poco evidente. En general se observa una abundancia de $\mathrm{Na}^{+}$respecto al $\mathrm{Cl}^{-}$, pero se considera que esa condición se arrastra desde el agua del río. $\mathrm{El} \mathrm{Cl}^{-}$es llamativamente alto en
3 muestras en el este y sureste, coincidiendo con la existencia de los establecimientos agrícolas más antiguos de la zona. Este aumento podría estar vinculado a prácticas de riego que fomentan la evaporación de agua en la zona no saturada y luego el lavado de sales. El $\mathrm{NO}_{3}{ }^{-}$también aumenta en los sectores agrícolas de mayor data.

El valor de CE más alto $(2.150 \mu \mathrm{S} / \mathrm{cm})$ se observa en el sector norte y estaría asociado a la disolución de sales desde sedimentitas (del Neógeno) de mayor cementación.

Los datos hidroquímicos en general corroboran la dinámica y el sentido de flujo, agregando además los efectos del factor antrópico mostrando el deterioro de la calidad del acuífero en los sectores de mayor actividad agrícola.

\section{Isotopía}

La composición de los isotopos estables, $\delta^{18} \mathrm{O}$ y $\delta^{2} \mathrm{H}$, muestra una baja dispersión en los datos correspondientes al agua subterránea, con valores entre $-138,4$ y $-130,1$ de $\delta^{2} \mathrm{H} \%$ o VSMOW; $y-18,29$ y $-16,96$ de $\delta^{18} \mathrm{O}^{1}{ }^{-}$ VSMOW (Figura 8). Estos valores serían comparables con la composición isotópica de las precipitaciones ocurridas por encima de los $2.500 \mathrm{msnm}$ (Hoke et al., 2013), dando una diferencia altitudinal entre la ubicación de los pozos de agua y dichas precipitaciones de más de 1.400 metros. El efecto de altitud (Dansgaard, 1964) en la provincia de Mendoza ha sido previamente determinado en los estudios de Panarello el al. (1993) y Panarello y Dapeña (1996).

En el otro extremo se encuentra la composición isotópica de la vertiente $\mathrm{La}$ Crucesita, $-50,4 \delta^{2} \mathrm{H}$ y $-8,06$ de $\delta^{18} \mathrm{O}$, la cual presenta una composición similar a las de las precipitaciones de la zona, indicando una recarga local para dicha vertiente. En lo que respecta al río Mendoza, presenta valores enriquecidos de $\delta^{2} \mathrm{H}$ y $\delta^{18} \mathrm{O}$ respecto al agua subterránea $-121,9 \delta^{2} \mathrm{H}$ y $-16,29 \delta^{18} \mathrm{O}$. La diferencia entre la composición isotópica del río Mendoza y el agua subterránea se puede asociar con el hecho de que la muestra del río es un valor puntual que corresponde al inicio de la primavera, mientras que las muestras de agua subterránea responden a una homogenización que ocurre a nivel acuífero y se ajustaría más a valores medios anuales de precipitaciones. Los valores del río también pueden responder a un aporte de aguas termales existentes inmediatamente aguas arriba del tramo muestreado. Esta última hipótesis es un punto a profundizar en futuros estudios.

\section{Discusión}

En zonas áridas y semiáridas la identificación de las zonas de recarga de acuíferos puede ser difícil de lograr, especialmente porque dados los déficit hídricos del área, la recarga puede ser alóctona proveniente de zonas remotas. 


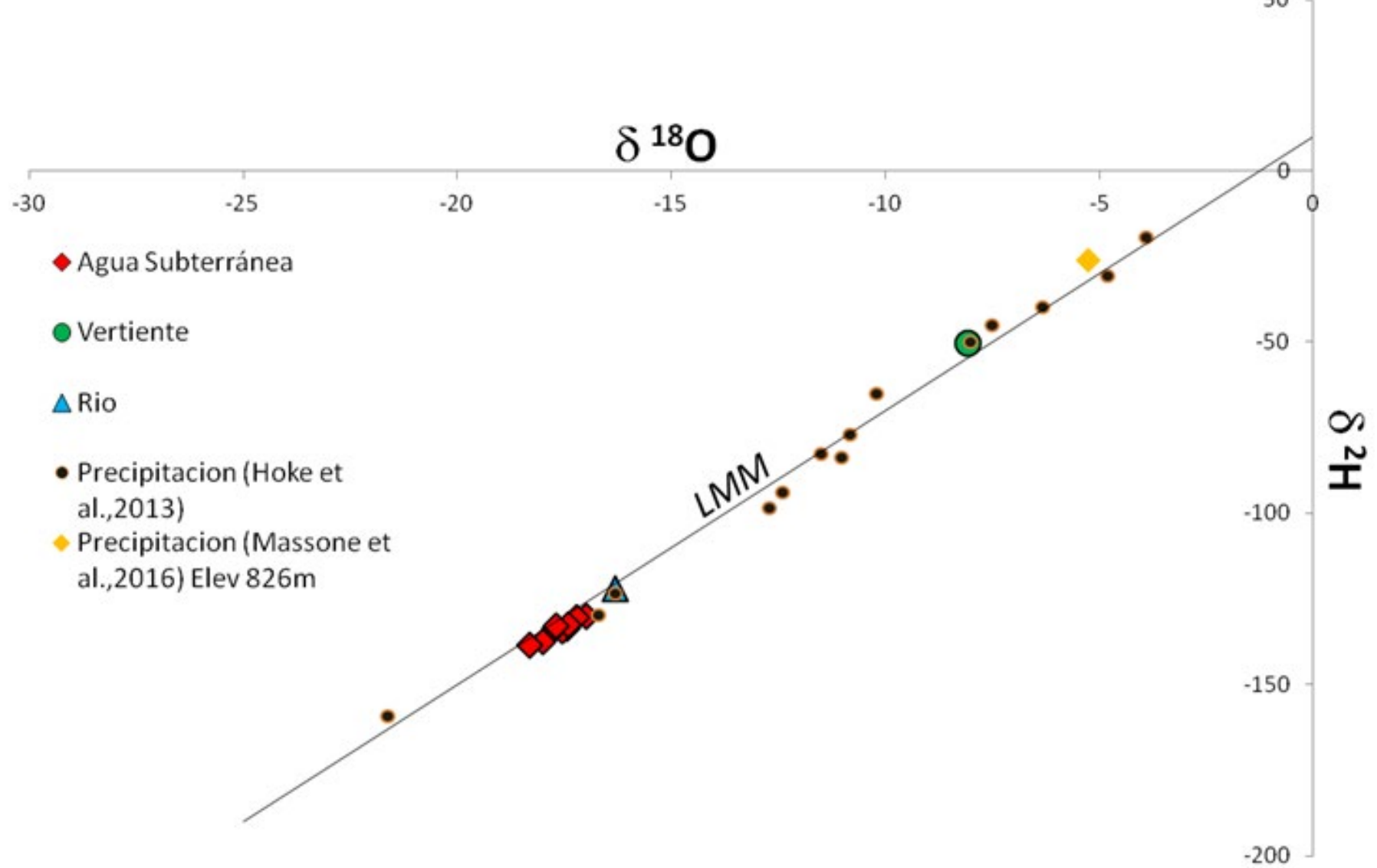

Figura 8. Diagrama $\delta^{2} \mathrm{H}$ vs. $\delta^{18} \mathrm{O}$ donde se representaron todas las muestras, la línea meteórica mundial-LMM (Craig,1961) y datos de precipitaciones (Hoke et al., 2013; Massone et al., 2016).

Figure 8. $\delta^{2} \mathrm{H}$ vs. $\delta^{18} \mathrm{O}$ diagram with all the samples, the world meteoric line-WML (Craig, 1961) and rainfall data (Hoke et al., 2013; Massone et al., 2016).

Las recargas desde cursos influentes con nacientes en zonas de excesos hídricos es un mecanismo frecuente. El análisis del comportamiento hidrológico a través de la piezometría permite usualmente reconocer zonas de recarga y la interacción entre ríos y acuíferos, sin embargo en zonas áridas especialmente, la disponibilidad y acceso a puntos de control de niveles suele ser escasa o estar concentrada en sectores particulares. El uso de trazadores hidroquímicos e isotópicos como aproximaciones a los sistemas de flujo es muy difundido, pero a la vez siempre es necesario partir de una base hidrogeológica conceptual.

En la región Norte de la provincia de Mendoza, la recarga desde el río Mendoza ha sido determinada mediante técnicas isotópicas por Panarello y Dapeña (1996), y también con un análisis que integra diferentes aproximaciones hacia el este de la zona de estudio realizado por Gómez et al. (2014). Un estudio amplio aguas arriba de la zona de estudio ha determinado diferentes composiciones de aguas de recarga (Crespo et al.,2016). Sin embargo en la zona de estudios, al realizar un análisis con más detalle se observa que las isopiezas indican una zona de carácter marcadamente influente del río Mendoza en la parte oriental, pero que hacia el oeste, más cercano a la Precordillera, las líneas de flujo subterráneo parecen desconectadas de la influencia del río y siguen una trayectoria noroeste-sureste. Por otra parte el mapa piezométrico permite también identificar líneas de flujo desde el oeste, en la zona denominada Cerrillada.

Estos sistemas de flujo pueden difícilmente ser cuantificados debido las imprecisiones en el conocimiento hidrodinámico, pero el uso de trazadores aporta en términos semi-cuantitativos. Los isótopos estables diferencian entre aguas de composición empobrecida, con fuentes posiblemente a unos 2500 metros de altura, que son tanto las agua subterráneas recargadas desde el río Mendoza, de las aguas del sector de la Precordillera que son isotópicamente mas enriquecidos, con diferencias altamente significativas entre ambos. La diferenciación que no puede hacerse con los datos isotópicos puede aproximarse con la hidroquímica. Por una parte nuevamente las aguas de la zona noroeste se diferencian por su tipo bicarbonatado y un mas bajo contenido de cloruros. Dentro de las aguas isotópicamente empobrecidas, es posible analizar los contenidos de cloruros en las líneas de flujo de la figura 4. El río Mendoza ingresa 
al sector estudiado con una concentración de cloruro cercana a los $100 \mathrm{mg} / \mathrm{l}$, pero la línea de flujo que viene desde la Precordillera y pasa por debajo del río sin conexión tiene en ese punto un valor de entre $60 \mathrm{y}$ $70 \mathrm{mg} / \mathrm{l}$ de cloruro. Esa línea de flujo luego alcanza los $100 \mathrm{mg} / \mathrm{l}$ de cloruro mas aguas abajo por mayor tiempo de tránsito. En cambio las aguas subterráneas cercanas al río en el sector oriental tiene los $100 \mathrm{mg} / \mathrm{l}$ de cloruro cerca del río manifestando su recarga desde el mismo. Si bien las aguas son en ambos casos sulfatadas cálcicas como resultado de la disolución de yesos de los sedimentos cuaternarios y de formaciones existentes en la cuenca superior (Gómez et al, 2014, Massone et al., 2016 y Crespo et al., 2016), es probable que el río Mendoza tenga mayor contenido de cloruros como resultado de aportes antrópicos y cierta concentración en el dique Potrerillos.

De esta forma el análisis de las líneas de flujo principales a partir de la piezometría permite identificar diferentes mecanismos de recarga y caracterizarlos como: a) agua subterránea desde Precordillera: isotópicamente enriquecida por recarga a partir de lluvias locales y con concentración de cloruro del orden de 40 a $70 \mathrm{mg} / \mathrm{l} \mathrm{b)} \mathrm{agua} \mathrm{del} \mathrm{río} \mathrm{Mendoza} \mathrm{isotópicamente} \mathrm{em-}$ pobrecida por nacientes a más de $2500 \mathrm{~m}$ de altura y con concentración de cloruro del orden de $100 \mathrm{mg} / \mathrm{l} ; \mathrm{c}$ ) agua desde la cerrillada, isotópicamente empobrecida y con concentración de cloruros entre 50 y $60 \mathrm{mg} / \mathrm{l}$.

Estos tres tipos de agua de aporte al acuífero en la zona de Agrelo se pueden usar como miembros extremos para la composición de las aguas subterráneas. Sin embargo existen limitaciones en su aplicación para cuantificar los aportes, por cuanto el cloruro también se incrementa en el agua subterránea por el mayor tiempo de contacto con el acuífero, pero su uso en el marco de las líneas de flujo determinadas por la piezometría contribuyen a establecer las principales condiciones hidrodinámicas del acuífero.

\section{Conclusiones}

La piezometría indica la existencia de varias zonas de recarga, una principal asociada al río Mendoza, una en el noroeste del área asociada a la Precordillera y una tercera zona en el oeste asociada a la Cerrillada Cacheuta-Tupungato. A partir de la hidroquímica e isotopía se puede determinar que, de todas esas zonas de recarga, la que tiene mayor incidencia en el acuífero es la vinculada al río. La dinámica de flujo determinada a partir de la medición de niveles estáticos es confirmada tanto por la hidroquímica como por los valores de transmisividad relativos calculados a partir de datos de caudales específicos.

Los datos isotópicos indican que el acuífero ha sido preferentemente alimentado por aguas empobrecidas isotópicamente respecto a los valores de la vertiente y las precipitaciones locales; se correlaciona con la composición isotópica de precipitaciones ocurridas a mayor altitud, confirmando el carácter mayormente alóctono de las aguas de recarga. Se observa también una variación entre la firma isotópica del Río Mendoza al momento del muestreo y la firma del acuífero, sugiriendo la ocurrencia de variaciones estacionales en la isotopía del río, o una característica particular del tramo muestreado. El agua subterránea muestra baja dispersión en su composición isotópica, probablemente debido a un mayor grado de mezcla.

\section{Agradecimientos}

Este trabajo se llevó a cabo en el marco del Curso Internacional de Hidrología Subterránea dictado por la FCIHS (Universidad Politécnica de Cataluña) y el ILLHA (Universidad Nacional del Centro de la Prov. de Buenos Aires). Los autores agradecen al proyecto internacional ARG/7/008, y al Organismo Internacional de Energía Atómica por el financiamiento, al Laboratorio de Análisis químicos del ILLHA, al Laboratorio de Hidrología Isotópica del Instituto de Geología de Costas y del Cuaternario de la Universidad de Mar del Plata y al Dr. Fidel Rivera de la FCIHS de Barcelona.

\section{Referencias}

Custodio, E. y Llamas, M. 1983. Hidrología Subterránea. Tomos I y II. Ediciones Omega, S.A. Casanova, 220. Barcelona. 1157 y $2350 \mathrm{pp}$.

Crespo, S., Aranibar, J., Gomez, L., Schwikowski, M., Bruetsch, S., Cara, L., \& Villalba, R. 2016. Ionic and stable isotope chemistry as indicators of water sources to the Upper Mendoza River basin, Central Andes of Argentina. Hydrological sciences journal, 62(4): 588-605.

Dansgaard, W. 1964. Stable isotopes in precipitation. Tellus, 16(4), pp.436-468.

Elango, L. and Kannan, R. 2007. Rock-water interaction and its control on chemical composition of groundwater. In: Dibyendu Sarkar et al.(ed). Concepts and Applications in Environmental Geochemistry. Developments in environmental science, 5, pp.229-243.

Foster, S. y Garduño, H. 2005. Argentina: Enfoque de Gestión Integrada para la Conservación del Agua Subterránea en los Acuíferos de Mendoza. Casos Esquemáticos. GW MATE Case profile collection. Banco Mundial. $16 \mathrm{pp}$

Giaccardi, A., Aguilera, D., Grizas, P. y Tognoli, P. 2018. Determinación de la velocidad de flujo del acuífero libre utilizando oscilaciones del nivel freático, Abanico de Agrelo, Mendoza, Argentina. $X$ Congreso Argentino de Hidrogeología, Salta. 7 pp.

Giambiagi, L., Ramos V.A., Godoy, E., Alvarez, P. y Orts, S. 2003. Cenozoic deformation and tectonic style of the Andes, between $33^{\circ}$ and $34^{\circ}$ South latitude. Tectonics, Vol.22(4), 1041.

Gómez, M.L., Aranibar,J., Wuillud,R., Rubio,C., Martinez,D.E., Soria,D., Monasterio, R., Villagra, P. and Goiran, S. 2014. Hydrogeology and hidrogeochemical modeling in phreatic aquifer of NE Men- 
doza, Argentina. Journal of Iberian Geology. Vol. 40(3)2014:521-538.

Gonfiantini, R.1978. Standards for stable isotopes measurements in natural compounds. Nature 271:534-536

Hernández, J., Martinis, N. y Fornero, L. 2012. Modelación Hidrológica de la Cuenca Norte de Mendoza. IT-146 CRA. Instituto Nacional del Agua, . Mendoza. $118 \mathrm{pp}$.

Hoke G., Aranibar J., Viale M., Araneo D., y Llano C. 2013. Seasonal moisture sources and the isotopic composition of precipitation, rivers, and carbonates across the Andes at $32.5-35.5^{\circ} \mathrm{S}$, Geochem. Geophys. Geosyst.,14, 962-978.

Ibañez, S., Di Lello, F., Euillades, P., Rovira, S., Fernandez, J., Quiroga, C., Ferrer, L, Sebok, A. y Chauvet, A. 2013. Cambios en los niveles piezométricos de la zona de recarga del acuífero de la cuenca norte después del dique Potrerillos, Mendoza. CONAGUA, San Juan. Argentina.

Lis, G., Wassenaar, L.I., Hendry, M.J. 2008. High-pre- cision laser spectroscopy $\mathrm{D} / \mathrm{H}$ and 180/160 measurements of microliter natural water samples. Anal Chem. 80:287-293

Massone, H., Martinez, D., Vich, A., Quiroz Londoño, M, Trombotto, D. y Grondona, S. 2016. Snowmelt contribution to the sustainability of the irrigated Mendoza's Oasis, Argentina: an isotope study. Environ Earth Sci (2016) 75:520. $11 \mathrm{pp}$.

Panarello, H., Dapeña, C. 1996. Mecanismos de recarga y salinización en las Cuencas de los Ríos Mendoza y Tunuyán, República Argentina. / XII Congreso Geológico de Bolivia, Tarija. 2, 1-18.

Panarello, H., Dapeña, C., García, E., Álvarez, A. 1993. Estudio isotópico e hidroquímico de los acuíferos de la Cuenca Norte mendocina, Ríos Mendoza yTunuyán. / XII Congreso Geológico Argentino, Mendoza. 4, 197-207.

Valero, C. 1993. Cuenca hidrogeológica de Mendoza Norte. Hidrogeología del área de máxima recarga del río Mendoza. / XII Congreso Geológico Argentino, Mendoza, 4, 155-165. 